\title{
ON THE STRUCTURE OF CERTAIN SUBALGEBRAS OF A UNIVERSAL ENVELOPING ALGEBRA $\left({ }^{1}\right)$
}

\author{
BY \\ BERTRAM KOSTANT AND JUAN TIRAO
}

\begin{abstract}
The representation theory of a semisimple group $G$, from an algebraic point of view, reduces to determining the finite dimensional representation of the centralizer $U^{\mathfrak{l}}$ of the maximal compact subgroup $K$ of $G$ in the universal enveloping algebra $U$ of the Lie algebra $\&$ of $G$. The theory of spherical representations has been determined in this way since by a result of Harish-Chandra $U^{t}$ modulo a suitable ideal $I$ is isomorphic to the ring of Weyl group $W$ invariants $U(a){ }^{W}$ in a suitable polynomial ring $U(a)$. To deal with the general case one must determine the image of $U^{t}$ in $U(t) \otimes U(a)$, where $t$ is the Lie algebra of $K$. We prove that if $W$ is replaced by the KunzeStein intertwining operators $\tilde{W}$ then $U^{t}$ suitably localized and completed is indeed isomorphic to $U(t) \otimes U(a) \widetilde{W}$ suitably localized and completed.
\end{abstract}

1. Introduction. Let $\boldsymbol{g}$ be a real semisimple Lie algebra and let $g=\mathfrak{l}+\mathfrak{p}$ be a Cartan decomposition of 8 . If $G$ is a Lie group, say with finite center, with Lie algebra $g$, it is known that many of the fundamental questions concerning the infinite dimensional representation theory of $G$ reduce to questions about the structure and finite dimensional representation theory of the algebra $G^{\mathfrak{l}}$. Here $G$ is the universal enveloping algebra, over $\mathbf{C}$, of $\mathbf{g}$ and $G^{l}$ is the centralizer of $\boldsymbol{l}$ in G. Briefly, the reason for this is as follows (Theorem of Harish-Chandra): To any quasi-simple irreducible Banach space representation $\pi$ of $G$ there is associated an algebraically irreducible $G$-module $V$ which is locally finite for $f$ and which determines $\pi$ up to infinitesimal equivalence. In fact one has a primary decomposition $V$ $=\bigoplus V_{\delta}$, where the sum is taken over the set $\hat{\mathrm{f}}$ of all equivalence classes $\delta$ of finite dimensional irreducible $\mathrm{f}$-modules, and the multiplicity of $\delta$ is finite for any $\delta \in \hat{\mathfrak{t}}$. Then, in particular, any $V_{\delta}$ is finite dimensional and hence, a finite dimensional $G^{\mathfrak{l}}$-module. The point is that $V$ itself as a $G$-module is completely determined by $V_{\delta}$ as a $G^{p}$-module for any fixed $\delta$ when $V_{\delta} \neq 0$. See Lepowsky and McCollum [10] and Lepowsky [9] for a nice exposition of this. See also Dixmier [3].

Received by the editors April 25, 1975.

AMS (MOS) subject classifications (1970). Primary 17Bxx, 17B10, $17 \mathrm{B35}$.

Key words and phrases. Universal enveloping algebra, intertwining operators, Weyl group, centralizer of the maximal compact subgroup, valuation on enveloping algebra.

( $\left.{ }^{1}\right)$ This paper is partially supported by NSF Grant No. GP-28969. Guggenheim Fellow; partially supported by NSF GP-42020X. 
If $V_{\delta_{0}} \neq 0$, where $\delta_{0}$ is the class of the trivial representation of $\mathfrak{l}$, then $\pi$ is called spherical. The approach above has been quite successful in dealing with spherical irreducible representations of $G$ (see e.g. Kostant [6]). Indeed, we may take $\delta=\delta_{0}$ and thus we have only to consider a quotient $G^{l} / I$ instead of $G^{\mathfrak{l}}$. Here $I$ is the intersection of $G^{l}$ with the left ideal in $G$ generated by $l$. Now by a theorem of Harish-Chandra, $G^{\mathbb{P}} / I$ is not only commutative but also isomorphic to a polynomial ring in $r$ variables where $r$ is the split rank of $G$. More precisely one has an algebra exact sequence

$$
0 \rightarrow I \rightarrow G^{p} \stackrel{\gamma}{\longrightarrow} A^{\widetilde{w}} \rightarrow 0
$$

where $\mathfrak{a}$ is a maximal abelian subalgebra of $\mathfrak{p}, A \subset G$ is the universal enveloping algebra of $a$ (over $C$ ) and $A^{\widetilde{w}}$ is the ring of $\widetilde{w}$-invariants in $A, \widetilde{w}$ being the translated Weyl group.

To investigate the general (not necessarily spherical) case along these lines one must look at $G^{\mathfrak{l}}$ itself, not just $G^{\mathfrak{l}} / \mathbf{I}$. It is known (see e.g. Lepowsky [9]) that the map (1.1) may be replaced by an exact sequence (see Proposition 3.1)

$$
0 \rightarrow G^{P} \stackrel{P}{\longrightarrow} K^{M} \rightarrow \mathrm{A}
$$

where $K$ is the universal enveloping algebra, over $\mathbf{C}$, of $\mathrm{l}, M$ is the centralizer of $a$ in the analytic subgroup $K$ of $G$ with Lie algebra $\mathfrak{l}, K^{M}$ is the centralizer of $M$ in $K$ and $K^{M} \otimes A$ is given the tensor product algebra structure. Moreover $P$ is an antihomomorphism of algebras. In order to generalize (1.1) it is necessary to determine the image of $P$. Towards the end we introduce the subalgebra $B$ of all elements in $K^{M} \otimes A$ which commute with certain intertwining operators. Such operators are in $1: 1$ correspondence with the elements of the Weyl group $W$ and are rather closely related to the operators considered in [12] and also to those studied in [8] and [5]. To define $B$ we consider $K^{M} \otimes A$ as a subalgebra of a larger algebra. In fact the relation of $B$ to $K^{M} \otimes A$ may be taken as the generalization of the relation of $A^{\widetilde{W}}$ to $A$.

A result of Tirao shows that the image of $P$ lies in $B$ (Theorem 3.2). However, unlike (1.1), $P$ is not an anti-isomorphism of $G^{l}$ onto $B$. But now we isolate an element $\gamma$ in the center of $G$ (hence in the center of $G^{l}$ ). One notes the mapping $P$ extends to an exact sequence

$$
0 \rightarrow G_{\gamma}^{l} \stackrel{P_{\gamma}}{\longrightarrow} B_{\gamma_{0}}
$$

where $G_{\gamma}^{l}$ is the localization of the ring $G^{l}$ with respect to $\gamma$ and $B_{\gamma_{0}}$ is the localization of $B$ with respect to $\gamma_{0}=P(\gamma)$.

Now there are natural valuations (in the sense of ring theory) on $G_{\gamma}^{f}$ and $B_{\gamma_{0}}$ so that the extended map $P_{\gamma}$ is compatible with these valuations. Thus $P_{\gamma}$ 
extends to a map $P_{\Gamma}$ of the respective completions $G_{\Gamma}^{l}$ and $B_{\Gamma_{0}}$. Our main result is the following:

THEOREM. The map $P_{\Gamma}: G_{\Gamma}^{l} \rightarrow B_{\Gamma_{0}}$ is a surjective anti-isomorphism.

2. Let $G$ be a noncompact connected semisimple Lie group with Lie algebra $g$; assume that $G$ has finite center. Let $g=\mathfrak{l}+\mathfrak{p}$ be a Cartan decomposition of $g$. Let $a$ be a Cartan subalgebra of the symmetric pair $(g, f)$. If $\alpha$ is a root of $g$ with respect to $a$, we denote by $g^{\alpha}$ the corresponding root subspace. Fix a linear ordering on the dual of $a$ and set

$$
\mathfrak{n}=\sum_{\alpha>0} g^{\alpha} \text { and } \overline{\mathfrak{n}}=\sum_{\alpha>0} g^{-\alpha}
$$

Then $\boldsymbol{g}=\mathfrak{l}+\mathfrak{a}+\mathfrak{n}$ is an Iwasawa decomposition of $\mathfrak{g}$.

Let $K$ be the analytic subgroup of $G$ with Lie algebra $\mathfrak{l}$, so $K$ is a maximal compact subgroup of $G$, and let $A, N, \bar{N}$ be the analytic subgroups of $G$ corresponding to $a, n$ and $\bar{n}$ respectively. $G$ has the global Iwasawa decomposition $G=K A N$. For $x$ in $G$ we write $x=\kappa(x)(\exp H(x)) n$ with $\kappa(x) \in K, H(x) \in \mathfrak{a}$, $n \in N$. Let $M$ (resp. $M^{\prime}$ ) be the centralizer (resp. the normalizer) of $a$ in $K ; W=$ $M^{\prime} / M$ is a finite group, the Weyl group.

Let $a_{c}^{*}$ be the complex dual of 0 . The Weyl group $W$ operates on $a_{c}^{*}$ by

$$
\langle\bar{w}(\lambda), H\rangle=\left\langle\lambda, \operatorname{Ad}\left(w^{-1}\right) H\right\rangle, \quad \lambda \in a_{\mathrm{C}}^{*}, H \in \mathfrak{a},
$$

where $\bar{w}=w M, w \in M^{\prime}$. Let $\rho(H)=1 / 2 \operatorname{tr}(\operatorname{ad}(H) \mid \mathfrak{n})$ for $H$ in $\mathbf{a}$; in other words, $\rho$ is half the sum of the positive roots with multiplicities.

We shall consider a family $U^{\lambda}$ of continuous representations of $G$ parametrized by $\lambda \in a_{c}^{*}$ (which may be viewed as being induced from characters of $A N$ ) and realized on $L^{2}(K)$. Given $x$ in $G, U^{\lambda}(x)$ is defined by the prescription

$$
\left(U^{\lambda}(x) f\right)(k)=e^{-(\lambda+\rho) H\left(x^{-1} k\right)} \cdot f\left(\kappa\left(x^{-1} k\right)\right), \quad f \in L^{2}(K)
$$

(see Warner [14, p. 445]).

For $w \in M^{\prime}$, define $\bar{N}_{w}=\bar{N} \cap w^{-1} N w$. Clearly $\bar{N}_{w}$ depends only on the coset $\bar{w}=w M$. We introduce intertwining operators for the representations $U^{\lambda}$ by considering the formal integral (for the statement about convergence see Proposition 2.1 below).

$$
(A(w, \lambda) f)(k)=\int_{\bar{N}_{w}} e^{-(\lambda+\rho) H(v)} f(k w \kappa(v)) d v
$$

where $\lambda \in a_{\mathrm{C}}^{*}, f \in C^{\infty}(K)$ and the Haar measure $d v$ on $\bar{N}_{w}$ is normalized by (see Schiffmann [12, p. 35]),

$$
\int \bar{N}_{w} e^{-2 \rho(H(v))} d v=1
$$


If $\alpha$ is a root of $g$ with respect to $a$, we denote by $H_{\alpha}$ the unique element in a such that $\alpha(H)=B\left(H_{\alpha}, H\right)$ for $H \in a$ ( $B$ is the Killing form of $\mathbf{g}$ ).

We recall that every $\bar{w} \in W$ can be decomposed as the product of reflections with respect to the simple roots. The minimum integer $q$, such that there exist $q$ simple roots, not necessarily different, $\alpha_{1}, \ldots, \alpha_{q}$ with $\bar{w}=s_{\alpha_{1}} \cdots s_{\alpha_{q}}\left(s_{\alpha}=\right.$ reflection corresponding to $\alpha$ ) is by definition the length $l(\bar{w})$ of $\bar{w}$.

Let $C^{\infty}(K)$ denote the set of $C^{\infty}$ complex-valued functions on $K$ equipped with the usual Fréchet structure (Schwartz topology).

We come to Schiffmann's results which can be found in [12], except that Schiffmann deals with the "induced picture". We state them in the following proposition for future reference. One notes first that $C^{\infty}(K)$ is stable under the action of $U^{\lambda}(x), x \in G$.

Proposition 2.1. (i) The domain of convergence (absolute) of the intertwining integrals $(2.1)$ is the set $S(\bar{w})$ of all $\lambda \in a_{\mathrm{C}}^{*}$ such that $\operatorname{Re}\left(\lambda\left(H_{\alpha}\right)\right)>0$ for every positive root $\alpha$ such that $\bar{w}(\alpha)<0$.

(ii) If $\lambda \in S(\bar{w}), A(w, \lambda)$ is a continuous endomorphism of $C^{\infty}(K)$, and $U^{\bar{w}(\lambda)}(x) A(w, \lambda) f=A(w, \lambda) U^{\lambda}(x) f$ for all $x \in G, f \in C^{\infty}(K)$.

(iii) Let $w_{1}, w_{2} \in M^{\prime}$ such that $l\left(\bar{w}_{1} \bar{w}_{2}\right)=l\left(\bar{w}_{1}\right)+l\left(\bar{w}_{2}\right)$. Then $S\left(\bar{w}_{1} \bar{w}_{2}\right)$ $=S\left(\bar{w}_{2}\right) \cap \bar{w}_{2}^{-1} S\left(\bar{w}_{1}\right)$ and $A\left(w_{1} w_{2}, \lambda\right)=A\left(w_{1}, \bar{w}_{2}(\lambda)\right) A\left(w_{2}, \lambda\right)$ for $\lambda \in S\left(\bar{w}_{1} \bar{w}_{2}\right)$.

One uses this result to establish

Proposition 2.2. (i) Given $w \in M^{\prime}$, for $\lambda \in S(w)$ the linear form

$$
T(w, \lambda): f \mapsto \int_{\bar{N}_{w}} e^{-(\lambda+\rho) H(v)} f(w \kappa(v)) d v, \quad f \in C^{\infty}(K),
$$

defines a distribution on $K$.

(ii) Let $w_{1} w_{2} \in M^{\prime}$ such that $l\left(\bar{w}_{1} \bar{w}_{2}\right)=l\left(\bar{w}_{1}\right)+l\left(\bar{w}_{2}\right)$. Then

$$
T\left(w_{1} w_{2}, \lambda\right)=T\left(w_{1}, \bar{w}_{2}(\lambda)\right) * T\left(w_{2}, \lambda\right) \text { for } \lambda \in S\left(\bar{w}_{1} \bar{w}_{2}\right) \text {. }
$$

Proof. (i) follows from Proposition 2.1(ii) by observing that $\langle T(w, \lambda), f\rangle$ $=(A(w, \lambda) f)(e)$. To prove (ii) we note that

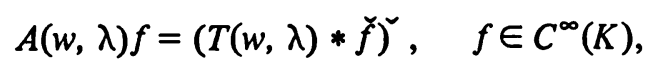

where $\check{f}$ denotes the function $k \mapsto f\left(k^{-1}\right)$. If $\lambda \in S\left(\bar{w}_{1} \bar{w}_{2}\right)$, Proposition 2.1(iii) gives

$$
A\left(w_{1} w_{2}, \lambda\right) f=A\left(w_{1}, \bar{w}_{2}(\lambda)\right) A\left(w_{2}, \lambda\right) f, \quad f \in C^{\infty}(K),
$$

which can be rewritten

$$
\begin{aligned}
& \left(T\left(w_{1} w_{2}, \lambda\right) * ð\right)^{\swarrow}=\left(T\left(w_{1}, \bar{w}_{2}(\lambda)\right) *\left(A\left(w_{2}, \lambda\right) f\right)^{\swarrow}\right)^{\swarrow} \\
& =\left(T\left(w_{1}, \bar{w}_{2}(\lambda)\right) * T\left(w_{2}, \lambda\right) * \check{f}\right)^{\swarrow} \text {. }
\end{aligned}
$$


Therefore,

$$
T\left(w_{1} w_{2}, \lambda\right) * \check{f}=T\left(w_{1}, \bar{w}_{2}(\lambda)\right) * T\left(w_{2}, \lambda\right) * \check{f} .
$$

Now evaluating at the identity $e$ and using the fact that $\langle T, f\rangle=(T * \check{f})(e)$, we complete the proof of the proposition.

3. Let $U$ be the universal enveloping algebra of the complexification $u_{c}$ of the Lie algebra $u$ of a Lie group $U$. As is well known, we may regard $U$ as the algebra of distributions on $U$ whose support is the identity $\{e\}$. One knows that $D \in U$ defines a left invariant differential operator $f \mapsto D f$ on $G$ where $D f=$ $f * \check{D}$ and $D \mapsto \check{D}$ is the usual antipode in $U$. One also knows that $\langle D, f\rangle=$ $[D f](e), f \in C_{c}^{\infty}(U)$.

Let $g_{C}, \mathfrak{l}_{C}, a_{C}, n_{C}$ be the complexifications of $\mathfrak{g}, \mathfrak{l}, \mathfrak{a}, \mathfrak{n}$, respectively. We denote by $G$ the universal enveloping algebra of $8_{\mathrm{C}}$, and by $K, A$ and $N$, the universal enveloping algebras of $\mathfrak{l}_{\mathbf{C}}, \mathfrak{a}_{\mathbf{C}}$ and $\mathfrak{n}_{\mathbf{C}}$, respectively, regarded as canonically embedded in $G$. We have

$$
G=K A N=K A\left(C 1+N n_{C}\right)=K A+G n_{\mathbf{C}} .
$$

Let $P: G \rightarrow K A$ denote the corresponding projection map. We give $K A$ an algebra structure by identifying it with the algebra $K \otimes A$, and we also regard $P$ as a map $P: G \rightarrow K \otimes A$. Let $G^{K}$ and $K^{M}$ denote the centralizers of $K$ in $G$ and of $M$ in $K$, respectively. A proof of the following proposition can be found in Lepowsky [9].

Proposition 3.1. P defines an injective antihomomorphism of $G^{K}$ into $K^{M} \otimes A$.

The algebra $A$ is just the symmetric algebra $S\left(a_{C}\right)$; hence each linear mapping $\lambda: a \rightarrow C$ extends uniquely to a homomorphism $D \rightarrow D(\lambda)$ of $A$ into $\mathbf{C}$ satisfying $1(\lambda)=1$. Now given $\lambda \in a_{C}^{*}$ we can also consider the homomorphism $K \otimes A \rightarrow K$ defined by $E \otimes D \mapsto(E \otimes D)(\lambda)=D(\lambda) E(E \in K, D \in A)$.

We take the opportunity to prove the following unpublished result of Tirao.

THEOREM 3.2. Given $w \in M^{\prime}, \lambda \in S(\widehat{w})$, we have

$$
T(w, \lambda) * P(D)(-\lambda-\rho)=P(D)(-\bar{w}(\lambda)-\rho) * T(w, \lambda) \text { for all } D \in G^{K} \text {. }
$$

Proof. Consider the following identity

$$
\begin{aligned}
& e^{-(\bar{w}(\lambda)+\rho) H(x)} \int_{\bar{N}_{w}} e^{-(\lambda+\rho) H(v)} f(\kappa(x) w \kappa(v)) d v \\
& =\int_{\bar{N}_{w}} e^{-(\lambda+\rho)(H(v)+H(x w \kappa(v)))} f(\kappa(x w \kappa(v))) d v, \quad x \in G,
\end{aligned}
$$


which is another way of writing $\left(U^{\bar{w}(\lambda)}\left(x^{-1}\right) A(w, \lambda) f\right)(e)=\left(A(w, \lambda) U^{\lambda}\left(x^{-1}\right) f\right)(e)$ (cf. Proposition 2.1(ii)). Let $\varphi_{1}(x)$ and $\varphi_{2}(x)$ denote the left- and right-hand sides of (3.1), respectively. It is also convenient to introduce the following notation: given $\lambda \in a_{c}^{*}$ and $f \in C^{\infty}(K)$, let

$$
F_{f}^{\lambda}(x)=e^{-(\lambda+\rho) H(x)} f(k(x)), \quad x \in G .
$$

Then since $H(x n)=H(x)$ and $\kappa(x n)=\kappa(x)$ for $n \in N$ it follows that $D F_{f}^{\lambda}$ $=0$ for $D \in G \mathfrak{n}_{\mathrm{c}}$, i.e., $D F_{f}^{\lambda}=P(D) F_{f}^{\lambda}$, for $D \in G$. Since $H(x \exp H)=H(x)+$ $H$ and $\kappa(x \exp H)=\kappa(x)(H \in \mathfrak{a})$, we have $D F_{f}^{\lambda}=D(-\lambda-\rho) F_{f}^{\lambda}$, for $D \in A$. Having in mind the decomposition $G=K A \oplus G n_{\mathrm{C}}$ it follows that $D F_{f}^{\lambda}=$ $P(D)(-\lambda-\rho) F_{f}^{\lambda}$, for $D \in G$.

If $f$ is a continuous function on $K$ we shall write $f^{R(k)}$ for the composite function $f \circ R(k)$ where $R(k)$ is a right translation by $k \in K$.

Given $D \in G$, we have

$$
\begin{aligned}
{\left[D \varphi_{1}\right](e) } & =\int_{\bar{N}_{w}} e^{-(\lambda+\rho) H(v)}\left[D F_{f^{R(w \kappa(v))}}^{\bar{w}(\lambda)}\right](e) d v \\
& =\int_{\bar{N}_{w}} e^{-(\lambda+\rho) H(v)}\left[P(D)(-\bar{w}(\lambda)-\rho) F_{f^{R(w \kappa(v))}}^{\bar{w}(\lambda)}\right](e) d v \\
& =\int_{\bar{N}_{w}} e^{-(\lambda+\rho) H(v)}\left[P(D)(-\bar{w}(\lambda)-\rho) f^{R(w \kappa(v))}\right](e) d v \\
& =\langle P(D)(-\bar{w}(\lambda)-\rho) * T(w, \lambda), f\rangle .
\end{aligned}
$$

Now let $D \in G^{K}$ and differentiate $\varphi_{2}$ to obtain

$$
\begin{aligned}
{\left[D \varphi_{2}\right](e) } & =\int_{\bar{N}_{w}} e^{-(\lambda+\rho) H(v)}\left[D F_{f}^{\lambda}\right](w \kappa(v)) d v \\
& =\int_{\bar{N}_{w}} e^{-(\lambda+\rho) H(v)}\left[P(D)(-\lambda-\rho) F_{f}^{\lambda}\right](w \kappa(v)) d v \\
& =\int_{\bar{N}_{w}} e^{-(\lambda+\rho) H(v)}[P(D)(-\lambda-\rho) f](w \kappa(v)) d v \\
& =\langle T(w, \lambda) * P(D)(-\lambda-\rho), f\rangle \text {. Q.E.D. }
\end{aligned}
$$

Given a finite dimensional irreducible representation $\left(V_{\delta}, \delta\right)$ of $K$ let us consider the maps

$$
P_{\delta}=(\delta \otimes 1) \circ P: G^{K} \rightarrow \operatorname{End}\left(V_{\delta}\right) \otimes A
$$

and

$$
p_{\delta}=(\operatorname{tr} \otimes 1) \circ P_{\delta}: G^{K} \rightarrow A
$$

When $\delta$ is the trivial one-dimensional representation of $K, P_{\delta}$ (or $p_{\delta}$ ) gives 
Harish-Chandra's famous homomorphism $\gamma: G^{K} \rightarrow A$. Theorem 3.2 generalizes that part of Harish-Chandra's theorem which asserts that the image of $\gamma$ is contained in the ring of $\widetilde{W}$-invariants of $A$ ( $\widetilde{W}$ denotes the translated Weyl group). One also has the following result of Lepowsky (see [9])

$$
\left.p_{\delta}(D) \lambda-\rho\right)=p_{\delta}(D)(\bar{w}(\lambda)-\rho)
$$

for all $D \in G^{K}, \bar{w} \in W, \lambda \in a_{C}^{*}$.

From Theorem 3.2 we get instead the more precise result

$$
\delta(T(w, \lambda)) P_{\delta}(D)(-\lambda-\rho)=P_{\delta}(D)(-\bar{w}(\lambda)-\rho) \delta(T(w, \lambda))
$$

for all $D \in G^{K}, w \in M^{\prime}, \lambda \in S(\bar{w})$.

Note that $\delta(T(w, \lambda))$ is given by the integral

$$
\delta(T(w, \lambda))=\int_{\bar{N}_{w}} e^{-(\lambda+\rho) H(v)} \delta(w \kappa(v)) d v, \quad \lambda \in S(\bar{w}) .
$$

Let $n=\operatorname{dim} \bar{N}_{w}$. From Theorem 4.1, it will follow that there exists a nonzero complex number $t_{w}(\lambda)$ such that

$$
\lim _{t \rightarrow+\infty} t^{n / 2} \delta(T(w, t \lambda))=t_{w}(\lambda) \delta(w), \quad w \in M^{\prime},
$$

uniformly on compact subsets of $S(\bar{w})$. Therefore, given any compact subset $\omega \subset S(\bar{w})$, for $t$ sufficiently large

$$
\delta(T(w, t \lambda)) \text { is invertible for all } \lambda \in \omega .
$$

Now it is clear that (3.3) implies (3.2).

Let $O(K)$ denote the space of distributions on $K$ equipped with the topology of uniform convergence on bounded subsets of $C^{\infty}(K)$. Let $O(K)^{M}$ be the centralizer of $M$ in $\mathcal{O}(K)$. We shall write $\delta_{k}$ for the Dirac measure at $k \in K$.

We can write

$$
T(w, \lambda)=\delta_{w} * T^{\prime}(\bar{w}, \lambda), \quad \lambda \in S(\bar{w}),
$$

(cf. Proposition 2.2(i)) where $T^{\prime}(\bar{w}, \lambda)$ is the distribution on $K$ defined by

$$
\left\langle T^{\prime}(\bar{w}, \lambda), f\right\rangle=\int_{\bar{N}_{w}} e^{-(\lambda+\rho) H(v)} f(\kappa(v)) d v, \quad \lambda \in S(\bar{w}), f \in C^{\infty}(K) .
$$

Now

$$
T^{\prime}(\bar{w}, \lambda) \in O(K)^{M} \quad \text { for } \lambda \in S(\bar{w}), \bar{w} \in W .
$$

In fact, for $\lambda \in S(\bar{w}), f \in C^{\infty}(K)$ and $m \in M$ we have

$$
\begin{aligned}
\left\langle\delta_{m} * T^{\prime}(\bar{w}, \lambda) * \delta_{m^{-1}}, f\right\rangle & =\int_{\bar{N}_{w}} e^{-(\lambda+\rho) H(v)} f\left(m \kappa(v) m^{-1}\right) d v \\
& =\int_{\bar{N}_{w}} e^{-(\lambda+\rho) H\left(m v m^{-1}\right)} f\left(\kappa\left(m v m^{-1}\right)\right) d v
\end{aligned}
$$


because $M$ normalizes $N$. But the Haar measure $d v$ of $\bar{N}_{w}$ is invariant under $v \mapsto m u m^{-1}$; therefore $\delta_{m} * T^{\prime}(\bar{w}, \lambda) * \delta_{m-1}=T^{\prime}(\bar{w}, \lambda)$, which proves (3.6).

A consequence of Theorem 3.2 is the following

Corollary 3.3. Assume $O(K)^{M}$ is abelian (which is precisely the case when $G$ is one of the following classical rank one groups: $\mathrm{SO}(n, 1)$ or $\mathrm{SU}(n, 1))$. Then

$$
\delta_{w} * P(D)(\lambda-\rho)=P(D)(\bar{w}(\lambda)-\rho) * \delta_{w}
$$

for all $w \in M^{\prime}, \lambda \in \mathfrak{a}_{\mathrm{C}}^{*}$ and $D \in G^{K}$.

PROOF. Let $\delta$ be any finite dimensional irreducible representation of $K$. From (3.3) and (3.5) we obtain

$$
\delta(w) \delta\left(T^{\prime}(\bar{w}, \lambda)\right) P_{\delta}(D)(-\lambda-\rho)=P_{\delta}(D)(-\bar{w}(\lambda)-\rho) \delta(w) \delta\left(T^{\prime}(\bar{w}, \lambda)\right)
$$

for $w \in M^{\prime}, \lambda \in S(\bar{w})$ and $D \in G^{K}$. But since $T^{\prime}(\bar{w}, \lambda)$ and $P(D)(-\lambda-\rho)$ are in $O(K)^{M}$ (cf. (3.6) and Proposition 3.1) we have

$$
\delta(w) P_{\delta}(D)(-\lambda-\rho) \delta\left(T^{\prime}(\bar{w}, \lambda)\right)=P_{\delta}(D)(-\bar{w}(\lambda)-\rho) \delta(w) \delta\left(T^{\prime}(\bar{w}, \lambda)\right) .
$$

Now because of (3.4) and (3.5) we obtain

$$
\delta(w) P_{\delta}(D)(-\lambda-\rho)=P_{\delta}(D)(-\bar{w}(\lambda)-\rho) \delta(w), \quad \lambda \in a_{C}^{*},
$$

which in turn implies our assertion. Q.E.D.

4. Let $B$ be the set of all elements $B \in K^{M} \otimes A$ such that

$$
T(w, \lambda) * B(-\lambda-\rho)=B(-\bar{w}(\lambda)-\rho) * T(w, \lambda)
$$

for all $w \in M^{\prime}$ and all $\lambda \in S(\bar{w})$. Clearly $B$ is a subalgebra of $K^{M} \otimes A$, and according to Theorem 3.2 it contains the image $P: G^{K} \rightarrow K^{M} \otimes A$. The principal objective now is to get information about the leading term of $B \in B$. The following theorem is needed and should be compared with results of Cohn [1].

THEOREM 4.1. Given $w \in M^{\prime}$ let $n=\operatorname{dim} \bar{N}_{w}$. For each $\lambda \in S(\bar{w})$ there exists a nonzero complex number $t_{w}(\lambda)$ such that

$$
\lim _{t \rightarrow+\infty} t^{n / 2} T(w, t \lambda)=t_{w}(\lambda) \delta_{w}
$$

uniformly on compact subsets of $S(\bar{w})$.

Proof. We shall show that it is sufficient to consider the case when $\bar{w}=s_{\alpha}$ is the reflection corresponding to a simple root $\alpha$. In fact, given $w \in M^{\prime}$ we can write $\bar{w}=s_{\alpha_{1}} \cdots s_{\alpha_{q}}$ where $q=l(\bar{w})$ and $\alpha_{j}(j=1, \ldots, q)$ are simple roots. We can find elements $w_{1}, \ldots, w_{q}$ in $M^{\prime}$ such that $\bar{w}_{j}=s_{\alpha_{j}}(j=1, \ldots, q)$ and $w=w_{1} \cdots w_{q}$. Now from Proposition 2.2(ii) it follows that 


$$
T(w, \lambda)=T\left(w_{1}, \bar{w}_{2} \cdots \bar{w}_{q}(\lambda)\right) * T\left(w_{2}, \bar{w}_{3} \cdots w_{q}(\lambda)\right) * \cdots * T\left(w_{q}, \lambda\right) .
$$

On the other hand if $n_{j}=\operatorname{dim} \bar{N}_{w_{j}}(j=1, \ldots, q)$ we have $n=n_{1}+\cdots+n_{q}$ (cf. [12, Proposition 1.3, p. 12]). Therefore, if we assume the theorem when $\bar{w}=s_{\alpha}, \alpha$ a simple root, we get

$$
\begin{aligned}
\lim _{t \rightarrow+\infty} & t^{n / 2} T(w, t \lambda) \\
& =\lim _{t \rightarrow+\infty} t^{n_{1} / 2} T\left(w_{1}, w_{2} \cdots w_{q}(t \lambda)\right) * \cdots * \lim _{t \rightarrow+\infty} t^{n_{q} / 2} T\left(w_{q}, t \lambda\right) \\
& =t_{w_{1}}\left(\bar{w}_{2} \cdots \bar{w}_{q}(\lambda)\right) \cdots t_{w_{q}}(\lambda) \delta_{w_{1}} * \cdots * \delta_{w_{q}}=t_{w}(\lambda) \delta_{w},
\end{aligned}
$$

uniformly on compact subsets of $S(\bar{w})$. We have used the joint continuity of the convolution which is a consequence of the compactness of $K$. Next we shall prove the case $\bar{w}=s_{\alpha}, \alpha$ is a simple root, thus completing the proof of the theorem.

Let $w \in M^{\prime}$ be such that $\bar{w}=s_{\alpha}$, where $\alpha$ is a simple root. The Lie algebra $\overline{\mathfrak{n}}_{w}$ of $\bar{N}_{w}=\bar{N} \cap \bar{w}^{\prime} N w$ is given by $\bar{n}_{w}=8^{-\alpha}+8^{-2 \alpha}$. Let $G_{\alpha}$ be the analytic subgroup whose Lie algebra is the smallest subalgebra of 8 containing $8^{-2 \alpha}, 8^{-\alpha}, 8^{\alpha}$ and $g^{2 \alpha}$. Then $G_{\alpha}$ is a semisimple Lie group with finite center. If we take $K_{\alpha}=G_{\alpha} \cap K, A_{\alpha}=G_{\alpha} \cap A$ and $N_{\alpha}=G_{\alpha} \cap N$ then $G_{\alpha}=K_{\alpha} A_{\alpha} N_{\alpha}$ is an Iwasawa decomposition of $G_{\alpha}$. The Lie algebra $a_{\alpha}$ of $A_{\alpha}$ is equal to $R H_{\alpha}$, i.e. $G_{\alpha}$ has real-rank one.

Let $p=\operatorname{dim} g^{-\alpha}$ and $q=\operatorname{dim} g^{-2 \alpha}$, then $\operatorname{dim} \bar{N}_{w}=p+q$. Since for $\lambda \in S(w), T(w, \lambda)=\delta_{w} * T^{\prime}(w, \lambda)$ (cf. 3.5) it is enough to establish that

$$
\lim _{t \rightarrow+\infty} t^{(p+q) / 2} T^{\prime}(\bar{w}, t \lambda)=t_{w}(\lambda) \delta_{e}
$$

$\left(0 \neq t_{w}(\lambda) \in C\right)$ uniformly on compact subsets of $S(w)$.

The distributions $T^{\prime}(w, \lambda)(\lambda \in S(\bar{w}))$ on $K$ come from the corresponding distributions on $K_{\alpha}$ (by restriction from $K$ to $K_{\alpha}$ ). This being a continuous map, the whole question reduces to the real-rank one group $G_{\alpha}$.

If $H_{\alpha}^{\prime}=2 \alpha\left(H_{\alpha}\right)^{-1} H_{\alpha}$, then $\rho\left(H_{\alpha}^{\prime}\right)=p+2 q$. Let $z=(p+2 q)^{-1} \lambda\left(H_{\alpha}^{\prime}\right)$; $\lambda$ is in $S(w)$ if and only if $\operatorname{Re} z>0$. We have to prove that given a compact subset $\omega$ of the set of all $z \in C$ with $\operatorname{Re} z>0$

$$
\lim _{t \rightarrow+\infty} t^{(p+q) / 2} \int_{\bar{N}_{w}} e^{-(t z+1) \rho(H(v))} f(\kappa(v)) d v=t_{w}(\lambda) f(e)
$$

uniformly for all $z \in \omega$ and all $f$ in each bounded subset of $C^{\infty}\left(K_{\alpha}\right)$.

We drop the subscript $\alpha$ and prove instead the following proposition which will complete the proof of Theorem 4.1.

Let $S_{\Delta}$ be the sector in the complex plane of all $z \in C$ such that $0<|z|$ $<\infty,|\arg z|<\pi / 2-\Delta$. 
Proposition 4.2. Let $G$ be a connected semisimple Lie group with finite center and real-rank one. Let $n=\operatorname{dim} \bar{N}$. Then, there exists a positive constant $c$ such that

$$
\lim z^{n / 2} \int_{\bar{N}} e^{-z \rho(H(v))} f(\kappa(v)) d v=c f(e)
$$

when $\left(z \rightarrow \infty, z \in S_{\Delta}, \Delta>0\right)$ uniformly for all $f$ in each bounded subset of $C^{\infty}(K)$.

First we need a few lemmas.

LEMmA 4.3. Let $\epsilon$ be a positive real number and $p$ a positive integer. Then

$$
\int_{0}^{\epsilon} r^{p-1}\left(1+r^{2}\right)^{-z} d r \sim 1 / 2 \Gamma(p / 2) z^{-p / 2} \quad\left(z \rightarrow \infty, z \in S_{\Delta}, \Delta>0\right) .
$$

Proof. The asymptotic behavior of the above integral can be established, for example, by Laplace's method, after introducing the new variable $t=$ $\log (1+r)^{2}$ (see Erdélyi [4, p. 37]), or we can proceed more directly as follows. Write

$$
\int_{0}^{\epsilon} r^{p-1}\left(1+r^{2}\right)^{-z} d r=\int_{0}^{\infty} r^{p-1}\left(1+r^{2}\right)^{-2} d r+g(z)
$$

We have

$$
\int_{0}^{\infty} r^{p-1}\left(1+r^{2}\right)^{-z} d r=\Gamma(p / 2) \Gamma(z-p / 2) / 2 \Gamma(z) \quad(\operatorname{Re} z>p / 2),
$$

which is asymptotic to $1 / 2 \Gamma(p / 2) z^{-p / 2}\left(z \rightarrow \infty, z \in S_{\Delta}, \Delta>0\right)$ (Stirling's formula; see Magnus [11, p. 12]).

On the other hand we can estimate $g(z)$ in the following way:

$$
|g(z)| \leqslant \int_{\epsilon}^{\infty} r^{p-1}\left(1+r^{2}\right)^{-\operatorname{Re} z} d r
$$

Given a positive real number $\delta$, there exists a positive number $A$ such that

$$
r^{p+1} \leqslant\left(\frac{1+r^{2}}{1+\delta}\right)^{\operatorname{Re} z} \quad \text { for } r \geqslant A, \operatorname{Re} z \geqslant p+1 .
$$

Now if we choose $\delta$ less than $\epsilon^{2}$, we can find another constant $B$ such that

$$
r^{p+1} \leqslant B\left(\frac{1+\epsilon^{2}}{1+\delta}\right)^{\operatorname{Re} z} \text { for } 0 \leqslant r \leqslant A, \operatorname{Re} z \geqslant p+1
$$

Therefore, there exists $C$ such that

$$
r^{p+1} \leqslant C\left(\frac{1+r^{2}}{1+\delta}\right)^{\operatorname{Re} z} \text { for } r \geqslant \epsilon, \operatorname{Re} z \geqslant p+1
$$

Hence $|g(z)| \leqslant C \epsilon^{-1}(1+\delta)^{-\operatorname{Re} z}$ for $\operatorname{Re} z \geqslant p+1$, which implies that $g(z)=$ $O\left(z^{-p / 2}\right)$ when $z \rightarrow \infty, z \in S_{\Delta}, \Delta>0$. This proves the lemma. Q.E.D. 
Let $B(\epsilon)(\epsilon>0)$ denote the set of all $(X, Y) \in \mathbf{R}^{p} \times \mathbf{R}^{q}$ such that $\|X\|$, $\|Y\| \leqslant \epsilon$.

Lemma 4.4. Let $p>0$. There exists a positive constant $c_{p, q}$ such that

$$
f(z)=\int_{B(\epsilon)}\left(\left(1+\|X\|^{2}\right)^{2}+\|Y\|^{2}\right)^{-z} d X d Y \sim c_{p, q^{z}}-(p+q) / 2
$$

when $z \rightarrow \infty, z \in S_{\Delta}, \Delta>0$.

Proof. We have to consider two different cases: (a) $q=0$ and (b) $q \neq 0$. Let $c_{n}$ be the Euclidean volume of the unit sphere in $\mathrm{R}^{n}$; in particular $c_{1}=2$.

(a) The usual formula for integration in polar coordinates yields

$$
f(z)=c_{p} \int_{0}^{\epsilon} r^{p-1}\left(1+r^{2}\right)^{-2 z} d r .
$$

The assertion follows from Lemma 4.3 with $c_{p, 0}=2^{-(1+p / 2)} \Gamma(p / 2) c_{p}$.

(b) In this case

$$
f(z)=c_{p} c_{q} \int_{0}^{\epsilon} \int_{0}^{\epsilon} r^{p-1} s^{q-1}\left(\left(1+r^{2}\right)^{2}+s^{2}\right)^{-z} d r d s .
$$

Letting for $0 \leqslant s \leqslant \epsilon, u=s\left(1+r^{2}\right)^{-1}$ we find

$$
\begin{aligned}
f(z) & =c_{p} c_{q} \int_{0}^{\epsilon} r^{p-1}\left(1+r^{2}\right)^{q-2 z} \int_{0}^{\epsilon\left(1+r^{2}\right)^{-1}} u^{q-1}\left(1+u^{2}\right)^{-z} d u d r \\
& =c_{p} c_{q} \int_{0}^{\epsilon} r^{p-1}\left(1+r^{2}\right)^{q-2 z} d r \int_{0}^{\epsilon} u^{q-1}\left(1+u^{2}\right)^{-z} d u-g(z)
\end{aligned}
$$

where

$$
g(z)=c_{p} c_{q} \int_{0}^{\epsilon} r^{p-1}\left(1+r^{2}\right)^{q-2 z} \int_{\epsilon\left(1+r^{2}\right)^{-1}}^{\epsilon} u^{q-1}\left(1+u^{2}\right)^{-z} d u d r .
$$

We can estimate $g(z)$ as follows:

$$
\begin{aligned}
|g(z)| & \leqslant c_{p} c_{q} \int_{0}^{\epsilon} r^{p-1}\left(1+r^{2}\right)^{q-2 \operatorname{Re} z} \int_{\epsilon\left(1+r^{2}\right)^{-1} u^{q-1}\left(1+u^{2}\right)^{-\operatorname{Re} z} d u d r}^{\epsilon} \\
& \leqslant c_{p} c_{q} \epsilon^{q+1} \delta\left(1+\delta^{2}\right)^{-\operatorname{Re} z} \int_{0}^{\epsilon} r^{p-1}\left(1+r^{2}\right)^{q-2 \operatorname{Re} z} d r
\end{aligned}
$$

where $\delta=\epsilon\left(1+\epsilon^{2}\right)^{-1}$. Therefore

$$
g(z)=O\left((\operatorname{Re} z)^{-p / 2}(1+\delta)^{-\operatorname{Re} z}\right)=O\left((1+\delta)^{-z}\right) \quad\left(z \rightarrow \infty, z \in S_{\Delta}, \Delta>0\right) .
$$

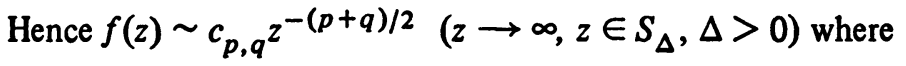

$$
c_{p, q}=2^{-(2+p / 2)} \Gamma(p / 2) \Gamma(q / 2) c_{p} c_{q} \text {. Q.E.D. }
$$

Proof of Proposition 4.2. Let $\alpha$ be the simple root of $g$ with respect to a. Then $\overline{\mathfrak{n}}=g^{-\alpha}+g^{-2 \alpha}$. Let $p=\operatorname{dim} g^{-\alpha}$ and $q=\operatorname{dim} g^{-2 \alpha}$. Let $Q$ be the quadratic form on $g$ defined by 


$$
Q(X)=4 B(X, \theta(X)) / B\left(H_{\alpha}^{\prime}, \theta\left(H_{\alpha}^{\prime}\right)\right)
$$

$\theta$ denotes the Cartan involution of $g$. If $v=\exp (X+Y), X \in \mathrm{g}^{-\alpha}, Y \in \mathrm{g}^{-2 \alpha}$, then (Helgason-Schiffmann, cf. [14, p. 38]) $H(v)=(a / 2) H_{\alpha}^{\prime}$ with $e^{2 a}=$ $(1+Q(X) / 2)^{2}+2 Q(Y)$. We make the identifications $g^{-\alpha} \simeq \mathbf{R}^{p}, g^{-2 \alpha} \simeq \mathbf{R}^{q}$ in such a way that $\|X\|^{2}=Q(X) / 2,\|Y\|^{2}=2 Q(Y)\left(X \in g^{-\alpha}, Y \in g^{-2 \alpha}\right)$. Then the integral under study can be written

$$
\mathrm{I}(z)=z^{(p+q) / 2} \int_{\mathbf{R}^{p} \times \mathbf{R}^{q}}\left(\left(1+\|X\|^{2}\right)^{2}+\|Y\|^{2}\right)^{-z b} f(\kappa(\exp (X+Y))) d X d Y
$$

where $b=(p+2 q) / 4$.

Let $B(\epsilon)=\left\{(X, Y) \in \mathbf{R}^{p} \times \mathbf{R}^{q}:\|X\|,\|Y\| \leqslant \epsilon\right\}$. We can write $\mathrm{I}(z)$ as the sum of an integral over $B(\epsilon)$ and an integral over $\mathbf{R}^{p} \times \mathbf{R}^{q}-B(\epsilon)$. Call the two resulting integrals $\operatorname{II}(\epsilon, z)$ and $\operatorname{III}(\epsilon, z)$ respectively. First of all we shall prove that $\operatorname{III}(\epsilon, z) \rightarrow 0$ as $z \rightarrow \infty, z \in S_{\Delta}, \Delta>0$, uniformly for all $f$ in a bounded subset of $C^{\infty}(K)$. There exists a constant $C$ such that the integrand of $\operatorname{III}(\epsilon, z)$ is bounded by

$$
C|z|^{(p+q) / 2}\left(\left(1+\|X\|^{2}\right)^{2}+\|Y\|^{2}\right)^{-b \operatorname{Rez}} .
$$

Given $d>0$, for $z \in S_{\Delta}$ and $|z|$ sufficiently large we have

$$
|z|^{(p+q) / 2} \leqslant\left(1+\epsilon^{2}\right)^{b \operatorname{Re} z-d} \leqslant\left(\left(1+\|X\|^{2}\right)^{2}+\|Y\|^{2}\right)^{b \operatorname{Re} z-d}
$$

whenever $(X, Y) \notin B(\epsilon)$. Therefore the integrand of $\operatorname{III}(\epsilon, z)$ is bounded by

$$
C\left(\left(1+\|X\|^{2}\right)^{2}+\|Y\|^{2}\right)^{-d}
$$

which is an integrable function for $d>b$ (see Wallach [13, p. 262]). By the dominated convergence theorem we have $\lim \operatorname{III}(\epsilon, z)=0$ when $z \rightarrow \infty, z \in S_{\Delta}$, $\Delta>0$ uniformly on bounded subsets of $C^{\infty}(K)$. In fact

$$
\lim |z|^{(p+q) / 2}\left(\left(1+\|X\|^{2}\right)^{2}+\|Y\|^{2}\right)^{-b \operatorname{Re} z}=0 \quad\left(z \rightarrow \infty, z \in S_{\Delta}, \Delta>0\right)
$$

if $(X, Y) \neq 0$.

Now consider $\operatorname{II}(\epsilon, z)$ and write

$$
\mathrm{II}(\epsilon, z)=f(e) z^{(p+q) / 2} \int_{B(\epsilon)}\left(\left(1+\|X\|^{2}\right)^{2}+\|Y\|^{2}\right)^{-z b} d X d Y+\mathrm{II}^{\prime}(\epsilon, z) .
$$

By Lemma 4.4 the first term tends to $c f(e)$ with $c=c_{p, q} b^{-(p+q) / 2}$ as $z \rightarrow \infty$, $z \in S_{\Delta}, \Delta>0$. Therefore to complete the proof of the proposition it is enough to show that given a bounded subset $B$ of $C^{\infty}(K)$ and a positive $\delta$, there exists a positive $\epsilon$ such that $\left|\mathrm{II}^{\prime}(\epsilon, z)\right|<\delta$ for $f \in B, z \in S_{\Delta}$ and $|z|$ sufficiently large. Now 


$$
\begin{aligned}
\left|I I^{\prime}(\epsilon, z)\right| \leqslant|z|^{(p+q) / 2} \int_{B(\epsilon)}\left(1+\|X\|^{2}+\|Y\|^{2}\right)^{-b \operatorname{Re} z} & \\
\cdot & |f(\kappa(\exp (X+Y)))-f(e)| d X d Y .
\end{aligned}
$$

From Lemma 4.4 it also follows that

$$
|z|^{(p+q) / 2} \int_{B(1)}\left(\left(1+\|X\|^{2}\right)^{2}+\|Y\|^{2}\right)^{-b \operatorname{Re} z} d X d Y
$$

is bounded in $S_{\Delta}$, say by a constant $A$. Given $\delta>0$ there exists $\epsilon(0<\epsilon \leqslant 1)$ such that

$$
|f(\kappa(\exp (X+Y)))-f(e)|<\delta A^{-1} \text { on } B(\epsilon)
$$

for all $f \in B$. Then $\left|\mathrm{II}^{\prime}(\epsilon, z)\right|<\delta$ for $z \in S_{\Delta}$ and $f \in B$, which completes the proof of Proposition 4.2. Q.E.D.

If $B \in K^{M} \otimes A$ we can view $B(\lambda)$ as a polynomial of degree $d$ on $a_{C}^{*}$ with coefficients in $K^{M}$. Let $B_{d} \in K^{M} \otimes A$ be the element such that $B_{d}(\lambda)$ is the leading term (homogeneous of degree $d$ in $\lambda$ ) of $B(\lambda)$. The Weyl group $W$ acts on $K^{M}$ and on $A$ via the adjoint representation, so we can define an action of $W$ on $K^{M} \otimes A$ by taking the tensor product action.

THEOREM 4.5. If $B \in B$ then the leading term $B_{d}$ of $B$ is W-invariant.

Proof. Given $w \in M^{\prime}$, let $n=\operatorname{dim} \bar{N}_{w}$. By hypothesis we have $T(w, \lambda) *$ $B(-\lambda-\rho)=B(-\bar{w}(\lambda)-\rho) * T(w, \lambda)$ for all $\lambda \in S(\bar{w})$. Now write

$$
t^{n / 2} T(w, t \lambda) * t^{-d} B(-t \lambda-\rho)=t^{-d} B(-\bar{w}(t \lambda)-\rho) * t^{n / 2} T(w, t \lambda)
$$

and let $t \rightarrow+\infty$. From Theorem 4.1 we obtain

$$
t(w, \lambda) \delta_{w} * B_{d}(-\lambda)=B_{d}(-\bar{w}(\lambda)) * t(w, \lambda) \delta_{w}, \quad \lambda \in S(\bar{w}),
$$

which proves our assertion. Q.E.D.

5. Let $g$ be a real semisimple Lie algebra and let $g=f+p$ be a Cartan decomposition. If subscript $\mathbf{C}$ denotes complexification one also has $g_{C}=$ $\mathfrak{t}_{\mathbf{c}}+\mathfrak{p}_{\mathbf{c}}$ :

Let $a$ be a maximal abelian subspace of $\mathfrak{p}$ and let $H \subseteq$ Aut( $\left.\mathfrak{p}_{\mathrm{C}}\right)$ be the analytic subgroup corresponding to $\operatorname{ad}_{p_{C}} \mathfrak{l}_{C} \subset \operatorname{End}\left(\mathfrak{p}_{C}\right)$. One knows that $x \in \mathfrak{p}_{C}$ is semisimple if and only if $x \in H \cdot a_{C}$ (see Kostant and Rallis [7, Theorem 1]).

Let $r=\operatorname{dim} a_{C}$. If $x \in p_{C}$ then one knows that $\operatorname{dim} p_{C}^{x} \geqslant r$ where one puts $\mathfrak{p}_{\mathbf{C}}^{x}=(\operatorname{Ker}$ ad $x) \cap \mathfrak{p}_{\mathbf{C}}$. An element $x \in \mathfrak{p}_{\mathbf{C}}$ is called regular if $\operatorname{dim} \mathfrak{p}_{\mathbf{C}}^{x}=r$. Sim. ilarly, let $\mathfrak{l}_{C}^{x}=(\operatorname{Ker}$ ad $x) \cap \mathfrak{l}_{C}$. Let $m$ be the centralizer of $a$ in $\mathfrak{l}$. One has $[7$, p. 770]

THEOREM 5.1. For any $x \in \mathfrak{p}_{\mathbf{C}}$ one has $\operatorname{dim} \mathfrak{l}_{\mathbf{C}}^{x}-\operatorname{dim} \mathfrak{p}_{\mathbf{C}}^{x}$ is independent of $x$. In particular $\operatorname{dim} \mathfrak{t}_{\mathbf{C}}^{x} \geqslant \operatorname{dim} \mathrm{m}$ and equality holds if and only if $x$ is regular in $\mathrm{P}_{\mathrm{C}}$. 
Now for any $x \in \mathfrak{p}_{\mathrm{C}},(\operatorname{ad} x)^{2}$ leaves $\mathfrak{p}_{\mathrm{C}}$ stable. Let $\alpha_{x}=\left.(\operatorname{ad} x)^{2}\right|_{\mathfrak{p}_{C}}$ and let $r_{x}$ be the multiplicity of the zero eigenvalue of $\alpha_{x}$.

Proposition 5.2. For all $x \in \mathfrak{p}_{\mathrm{C}}, r_{x} \geqslant r$.

PROof. Clearly $r_{x} \geqslant \operatorname{dim}\left(\operatorname{ker} \alpha_{x}\right) \geqslant \operatorname{dim} \mathfrak{p}_{\mathrm{C}}^{x} \geqslant$ r. Q.E.D.

Now we say that $x \in \mathfrak{p}_{\mathrm{C}}$ is s-regular if $r_{x}=r$.

Proposition 5.3. An element $x$ in $\mathfrak{p}_{\mathbf{c}}$ is s-regular if and only if $x$ is regular and semisimple.

PROOF. Assume $x \in \mathfrak{p}_{\mathrm{C}}$ is regular and semisimple. Then $\operatorname{ker} \alpha_{x}=\mathfrak{p}_{\mathrm{C}}^{x}$ because $x$ is semisimple, and $\operatorname{dim} \mathfrak{p}_{C}^{x}=r$ because $x$ is regular. Hence $x$ is $s$-regular.

Conversely, suppose $x$ is s-regular. Now if $y \in \mathfrak{p}_{\mathbf{C}}$ let $\boldsymbol{g}_{\mathbf{C}}^{0}(y)=\left\{u \in \mathbb{8}_{\mathbf{c}}\right.$ : $(\operatorname{ad} y)^{n} u=0$ for some $n$ ), and if $q=\min \left(\operatorname{dim}\left(g_{\mathrm{C}}^{0}(y) \cap \mathfrak{p}_{\mathrm{C}}\right)\right)$ over all $y \in \mathfrak{p}_{\mathrm{C}}$, we let $Q$ be the set of all $y \in \mathfrak{p}_{\mathbf{C}}$ such that $q=\operatorname{dim}\left(g_{\mathbf{C}}^{0}(y) \cap \mathfrak{p}_{\mathbf{C}}\right)$. Since $\operatorname{dim}\left(\boldsymbol{g}_{\mathbf{C}}^{0}(y)\right.$ $\cap \mathfrak{p}_{\mathrm{C}}$ ) is clearly the multiplicity of the zero eigenvalue of $\alpha_{y}$ it follows that $q=r$ and hence $x \in Q$. One knows that $Q=H \cdot\left(Q \cap a_{\mathrm{C}}\right)[7, \mathrm{p} .765]$ so the elements in $Q$ are semisimple. Hence $x$ is semisimple, and since it is clearly regular, we have completed the proof of the proposition. Q.E.D.

The theorem we wish to prove is

THEOREM 5.4. Let $x \in \mathfrak{p}_{\mathrm{C}}$ and let $l_{x}$ be the multiplicity of the zero eigenvalue of $\operatorname{ad} x$ in $b_{c}$. Then $l_{x} \geqslant l=\operatorname{dim} a+\operatorname{dim} m$ where equality holds if and only if $x$ is s-regular.

Proof. If $x$ is s-regular then one has (cf. Theorem 5.1) $\operatorname{dim} \mathbf{8}_{\mathrm{C}}^{x}=\operatorname{dim} \mathbf{P}_{\mathbf{C}}^{x}$ $+\operatorname{dim} \mathfrak{p}_{C}^{x}=\operatorname{dim} m+\operatorname{dim} \mathfrak{a}$ where $\mathbf{g}_{C}^{x}=\operatorname{ker} a d x$. However since $x$ is semisimple $\operatorname{dim} g_{C}^{x}$ is the multiplicity of the zero eigenvalue of ad $x$ in $8_{C}$ establishing the theorem in one direction.

Conversely assume $l_{x}=l$. For any $y \in \mathfrak{p}_{\mathrm{C}},(\operatorname{ad} y)^{2}$ leaves $\mathfrak{l}_{\mathrm{C}}$ stable. Let $d_{y}$ be the multiplicity of the zero eigenvalue of $(\operatorname{ad} y)^{2}$ in $t_{c}$. We have $l_{y}=d_{y}$ $+r_{y}, d_{y} \geqslant \operatorname{dim} t_{c}^{y} \geqslant \operatorname{dim} m$ and $r_{y} \geqslant r$. Also $l_{y}$ is equal to the multiplicity of the zero eigenvalue of $(\operatorname{ad} y)^{2}$ in $g_{c}$ as well as ad $y$. Therefore $l_{x}=l$ implies $r_{x}=r$ which concludes the proof of the theorem. Q.E.D.

For any vector space $V$, let $S(V)$ denote the symmetric algebra over $V$. For every nonnegative integer $i$, let $S(V)$ denote the homogeneous subspace of $S(V)$ of degree $i$.

Let $n=\operatorname{dim} g_{\mathrm{C}}$ and let $\mathrm{g}_{\mathrm{C}}^{\prime}$ be the dual of $g_{\mathrm{c}}$. Now for any $x \in \mathrm{g}_{\mathrm{C}}$ let $\operatorname{det}(t-\operatorname{ad} x)=\Sigma a_{i}(x) t^{i}$ be the characteristic polynomial of ad $x$. One has $a_{i} \in$ $\left(S^{n-i}\left(g_{\mathrm{C}}^{\prime}\right)\right)^{G}$ is an invariant polynomial where $G_{\mathrm{C}}$ denotes the adjoint group of $8_{C}$. Consider $a_{l}$. We then have 
Corollary 5.5. Let $x \in \mathfrak{p}_{\mathrm{c}}$. Then $a_{i}(x)=0$ for all $i<l$ and $a_{l}(x)=0$ if and only if $x \in \mathfrak{p}_{\mathrm{C}}$ is not s-regular.

Let $\mathfrak{p}_{\mathrm{C}}^{*} \subset \mathfrak{p}_{\mathrm{C}}$ denote the set of all s-regular elements in $\mathfrak{p}_{\mathrm{C}}$. Let $a=a_{l}$. Now let $b=\left.a\right|_{\mathfrak{p}_{\mathrm{C}}}$ so that $b \in S^{n-l}\left(\mathfrak{p}_{\mathrm{C}}^{\prime}\right)$, where $\mathfrak{p}_{\mathrm{C}}^{\prime}$ denotes the dual of $\mathfrak{p}_{\mathrm{C}}$. We note that $b \neq 0$ and in fact $p_{C}^{*}=\left\{x \in \mathfrak{p}_{\mathbf{C}}: b(x) \neq 0\right\}$. More explicitly if $\Delta$ is the set of roots, counting multiplicities of $\left(a_{c}, 8_{c}\right)$, then card $\Delta=n-l$ and $\left.b\right|_{a_{C}}=\Pi_{\alpha \in \Delta} \alpha$.

6. Now we regard $S\left(\mathfrak{p}_{\mathrm{c}}^{\prime}\right)$ as a subalgebra of $S\left(\boldsymbol{g}_{\mathrm{c}}^{\prime}\right)$ where if $f \in S\left(\mathfrak{p}_{\mathrm{c}}^{\prime}\right)$ then $f$ is also regarded as a function on $8_{\mathbf{C}}$ such that if $z \in 8_{\mathbf{C}}, z=x+y, x \in \mathfrak{l}_{\mathbf{C}}$, $y \in \mathfrak{p}_{\mathrm{C}}$ then $f(x+y)=f(y)$.

It follows that if $\boldsymbol{g}_{C}^{*}=\mathfrak{l}_{C}+p_{C}^{*}$ then $b \in S^{n-l}\left(g_{C}^{\prime}\right)$ and $g_{C}^{*}=\left\{z \in g_{C}\right.$ : $b(z) \neq 0\}$. That is, $8_{C}^{*}$ is an open affine subvariety of $8_{C}$ and the affine algebra of $\boldsymbol{g}_{\mathrm{C}}^{*}$ is the localization $S\left(\boldsymbol{g}_{\mathrm{c}}^{\prime}\right)_{b}$ of $S\left(g_{\mathrm{c}}^{\prime}\right)$ by $b$, so that $S\left(g_{\mathrm{c}}^{\prime}\right)_{b}$ is the ring of all rational functions on $\boldsymbol{g}_{\mathrm{C}}$ of the form $f / b^{k}$ where $f \in S\left(8_{\mathrm{C}}^{\prime}\right)$ and $k \in \mathbf{Z}$.

Now let $a_{c}^{*}=\left\{x \in a_{c}: \alpha(x) \neq 0\right.$ for all $\left.\alpha \in \Delta\right\}$; then $\mathfrak{t}_{\mathbf{c}}+a_{\mathbf{c}}^{*}=$ $\left\{z \in \mathfrak{t}_{\mathbf{c}}+a_{\mathbf{c}}: b_{0}(z) \neq 0\right\}$ where $b_{0}=b \mid \mathfrak{l}_{\mathbf{c}}+a_{\mathbf{c}}$. Thus $\mathfrak{t}_{\mathbf{c}}+\dot{a}_{\mathbf{C}}^{*}$ is an affine variety whose affine algebra is the localization $S\left(\left(\boldsymbol{l}_{c}+a_{c}\right)^{\prime}\right)_{b_{0}}$ of $S\left(\left(\mathfrak{l}_{c}+a_{c}\right)^{\prime}\right)$ by $b_{0}$. By now the injection map $\mathfrak{l}_{\mathbf{C}}+\boldsymbol{a}_{\mathbf{C}}^{*} \rightarrow \mathfrak{l}_{\mathrm{C}}+\mathfrak{p}_{\mathrm{C}}^{*}=\boldsymbol{b}_{\mathrm{C}}^{*}$ of affine varieties induces contravariantly the restriction homomorphism

$$
S\left(b_{c}^{\prime}\right)_{b} \rightarrow S\left(\left(\mathfrak{e}_{c}+a_{c}\right)^{\prime}\right)_{b_{0}}
$$

of affine algebras.

Now let $K_{\mathrm{C}}$ be the subgroup of $G_{\mathrm{C}}$ corresponding to ad $\mathfrak{t}_{\mathrm{C}}$. Then the affine variety $\mathrm{g}_{\mathrm{C}}^{*}$ is clearly stable under the action of the reductive algebraic group $K_{\mathrm{C}}$ and hence the ring of $K_{\mathrm{C}}$-invariants $A=S\left(8_{\mathrm{C}}^{\prime}\right)_{b}^{K} \mathbf{C}$ is an affine ring (finitely generated). Also if $M_{\mathrm{C}}^{\prime}$ is the normalizer of $a_{\mathrm{C}}$ in $K_{\mathrm{C}}$ then $M_{\mathrm{C}}^{\prime}$ is a reductive algebraic group operating on the affine variety $\boldsymbol{l}_{c}+a_{c}^{*}$ and hence $A_{0}=S\left(\left(\mathrm{f}_{\mathrm{C}}+a_{\mathrm{c}}\right)^{\prime}\right)_{b_{0}}^{M_{\mathrm{C}}^{\prime}}$ is also an affine ring. Since $M_{\mathrm{C}}^{\prime} \subset K_{\mathrm{C}}$ the homomorphism (6.1) restricted to $A$ induces a homomorphism

$$
\pi: A \rightarrow A_{0}
$$

We will prove the following theorem of Kostant.

THEOREM 6.1. The homomorphism $\pi: A \rightarrow A_{0}$ is an isomorphism of algebras.

We first establish some lemmas. Let $O$ be the set of all $K_{C}$ orbits in $8_{C}^{*}$ and let $O_{0}$ be the set of $M_{c}^{\prime}$ orbits in $\mathfrak{l}_{\mathbf{C}}+a_{\mathrm{c}}^{*}$.

LFmma 6.2. If $O \in O$ then $O \cap\left(\mathfrak{l}_{\mathrm{C}}+\mathrm{a}_{\mathrm{C}}^{*}\right)=O_{0}$ is an $M_{\mathrm{C}}^{\prime}$ orbit and the correspondence $O \mapsto O_{0}$ defines a bijection $\mathrm{O} \longrightarrow \mathrm{O}_{0}$. 
Proof. The only thing we really have to prove is that given $x, y \in O \cap$ $\left(\mathfrak{l}_{\mathrm{C}}+\mathrm{a}_{\mathrm{C}}^{*}\right)$ there exists $k \in M_{\mathrm{C}}^{\prime}$ such that $y=k \cdot x$. Write $x=x_{1}+x_{2}, y=$ $y_{1}+y_{2}$ where $x_{1}, y_{1} \in \mathbb{l}_{\mathrm{C}}$ and $x_{2}, y_{2} \in \mathfrak{a}_{\mathrm{C}}^{*}$. We know that there is $k \in K_{\mathrm{C}}$ such that $y=k \cdot x$ and therefore $y_{2}=k \cdot x_{2}$. Now we use the fact (see [7]) that if two elements in $a_{\mathbf{c}}$ are $K_{\mathbf{C}}$-conjugate then they are $M_{\mathbf{C}}^{\prime}$-conjugate. Hence there exists $m_{1} \in M_{\mathrm{C}}^{\prime}$ such that $y_{2}=m_{1} \cdot x_{2}$. Then $m_{1}^{-1} k \cdot x_{2}=x_{2}$. Since $x_{2}$ is s-regular $m_{1}^{-1} k=m$ centralizes $a_{C}$ (cf. [7, Lemma 20]). Thus $k=m_{1} m$ $\in M_{\mathbf{C}}^{\prime}$ and the lemma is proved.

LEMMA 6.3. With respect to the bijection $O \mapsto O_{0}$ of the previous lemma one has: $O$ is closed if and only if $O_{0}$ is closed.

Proof. Assume $O_{0}$ is closed and $x_{n} \rightarrow x, x_{n} \in O, x \in g_{C}^{*}$. Then by applying an element in $K_{\mathbf{C}}$ we may assume $x \in \mathfrak{l}_{\mathbf{C}}+\mathfrak{a}_{\mathbf{C}}^{*}$. Then we may find $k_{n} \in$ $K_{\mathrm{C}}, k_{n} \rightarrow e$ such that $k_{n} \cdot x_{n} \in \mathbb{P}_{\mathrm{C}}+\mathrm{a}_{\mathrm{C}}^{*}$ so that $k_{n} \cdot x_{n} \rightarrow x$. But $k_{n} \cdot x_{n}$ $\in O_{0}$ therefore $x \in O_{0}$. Hence $O$ is closed.

Proof of Theorem 6.1. We first observe that $\pi: A \rightarrow A_{0}$ is injective. Indeed if $0 \neq f \in A$ we must show $f \mid \mathfrak{l}_{\mathrm{C}}+a_{\mathrm{C}}^{*} \neq 0$. But if $f \mid \mathfrak{f}_{\mathrm{C}}+a_{\mathrm{C}}^{*}=0$ then $0=f \mid K_{\mathrm{C}} \cdot\left(\mathfrak{l}_{\mathrm{C}}+\mathrm{a}_{\mathrm{C}}^{*}\right)$. Thus $f=0$ since $K_{\mathrm{C}} \cdot\left(\mathfrak{l}_{\mathrm{C}}+\mathrm{a}_{\mathrm{C}}^{*}\right)=\mathbf{g}_{\mathrm{C}}^{*}$. Thus we may regard $A \subset A_{0}$. But now we assert: (1) $A$ is integrally closed in its quotient field $Q ;(2)$ if $\hat{A}_{0}$ (resp. $\hat{A}$ ) denotes the set of all homomorphisms $\chi: A_{0} \rightarrow \mathrm{C}$ (resp. $\chi: A \rightarrow \mathrm{C}$ ) then the map $\hat{A}_{0} \rightarrow \hat{A}, \chi \rightarrow \chi \circ \pi$ is a bijection.

To establish (1) we note that if $f \in Q$ satisfies a monic polynomial equation with coefficients in $A \subset S\left(g_{\mathrm{C}}^{\prime}\right)_{b}$ then $f \in S\left(g_{\mathrm{C}}^{\prime}\right)_{b}$ since $S\left(g_{\mathrm{C}}^{\prime}\right)_{b}$, a localization of a polynomial ring, is integrally closed. Because $f \in Q, f=a_{1} / a_{2}, a_{1}, a_{2} \in A$ and $a_{2} \neq 0$. Hence $f a_{2}=a_{1}$; applying $k \in K_{\mathrm{C}}$ we get $f^{k} a_{2}=a_{1}=f a_{2}$, therefore $f^{k}=f$, i.e. $f \in A$.

Now (2) follows from Lemma 6.3 since one knows that the natural map $0 \rightarrow \hat{A}$ and $O_{0} \rightarrow \hat{A}_{0}$ give a bijection between the set of all closed orbits in 0 and $\hat{A}$, and the set of all closed orbits in $O_{0}$ and $\hat{A}_{0}$, respectively. (See e.g. Dieudonné [2].) Now (2) implies that $\hat{A}_{0} \rightarrow \hat{A}$ is a bijective, birational map of affine varieties. But (1) implies that $\hat{A}$ is normal. Hence by Zariski's Main Theorem (see e.g. [15, p. 413]) the map $\hat{A}_{0} \rightarrow \hat{A}$ is an isomorphism and hence $A_{0}=A$. Q.E.D.

7. A valuation on a ring $R$ is a map $\nu: R \rightarrow Z \cup\{-\infty\}$ such that: (1) $\nu(r)=-\infty$ if and only if $r=0$, (2) $\nu(r+s) \leqslant \max (\nu(r), v(s)),(3) v(r s)=v(r)+$ $\nu(s)$. If $R_{n}=\{r \in R . v(r) \leqslant n\}$ then $R_{n} \subset R_{n+1}, \bigcap_{-\infty<n<\infty} R_{n}=\{0\}$ and $R_{n}(n \in \mathrm{Z})$ defines a system of neighborhoods of 0 and hence a topology on $R$. The valuation also defines a uniform structure on $R$ so that we may complete $R$ obtaining a ring $\bar{R}$. To each $\bar{r} \in \bar{R}$ there is a Cauchy sequence $r_{n} \in R$ such that $r_{n} \rightarrow \bar{r}$. If $r_{n} \rightarrow \bar{r}$ and $s_{n} \rightarrow \bar{s}$ then $r_{n} s_{n} \rightarrow \bar{r} \bar{s}, r_{n}+s_{n} \rightarrow \bar{r}+\bar{s}$. 
Now if $R$ is an integral domain and it satisfies the Ore condition (i.e.: $R a$ $\cap R b \neq\{0\}$ for all $a, b \neq 0)$ then $v$ extends to $Q(R)$, the left quotient division ring of $R$, by setting $\nu\left(a^{-1} b\right)=\nu(b)-\nu(a)$.

EXAMPLE. Let $\mathfrak{h}$ be a Lie algebra and $\mathfrak{i} \subset \mathfrak{b}$ any subalgebra in $\mathfrak{b}$. Let $J \subset$ $H$ be the corresponding universal enveloping algebras. Let $H_{(n)}$ be the usual filtration of $H$. Thus $H_{(n)}$ is spanned by $x_{1} \cdots x_{j}, x_{i} \in \mathfrak{h}, j \leqslant n$, and the identity. We claim that $\mathrm{JH}_{(n)}=\mathrm{H}_{(n)} \mathrm{J}$.

To prove, for example, that $J H_{(n)} \subset H_{(n)} J$ one notices that if $x_{1}, \ldots, x_{j}$ $\in \mathfrak{h}, y \in \mathfrak{i}$, then by induction

$$
\left.y x_{1} \cdots x_{j}=x_{1} \cdots x_{j} y+\sum_{i=1}^{j} x_{1} \cdots\left[y, x_{i}\right] \cdots x_{j} \in H_{(n)}\right]
$$

if $j \leqslant n$. Thus we get a new filtration of $H$ by putting $H_{n}=J H_{(n)}$ since now $H_{n} H_{m} \subset H_{n+m}$.

THEOREM 7.1. If $0 \neq a \in H$ let $v(a)=\min n$ such that $a \in H_{n}$ and let $\nu(0)=-\infty$. Then $v$ is a valuation on $H$.

Proof. Let $q$ be a linear complement of $\mathfrak{i}$ in $\mathfrak{b}$ so that $\mathfrak{b}=\mathfrak{i}+q$ (direct sum). Let $y_{1}, \ldots, y_{k}$ be a basis of $q$. Then

$$
H=\underset{\left(m_{1}, \ldots, m_{k}\right)}{\bigoplus} J y_{1}^{m_{1}} y_{2}^{m_{2}} \cdots y_{k}^{m_{k}}
$$

by the Birkhoff-Witt theorem. In fact, if $u \in H, u=\Sigma u_{m_{1}, \ldots, m_{k}}$ where $u_{m_{1}}, \ldots, m_{k} \in J y_{1}^{m_{1}} \cdots y_{k}^{m_{k}}$, then $v(u)=\max _{u_{m} \neq 0}|m|$ where $m=\left(m_{1}, \ldots, m_{k}\right)$, $|m|=\Sigma_{i=1}^{k} m_{i}$.

The only thing to be proved is that $\nu(a b)=\nu(a)+\nu(b)$ for all $a, b \in H$. Since clearly $\nu(a b) \leqslant \nu(a)+\nu(b)$ to prove the equality we may assume that $a=$ $\Sigma_{|m|=\nu(a)} a_{m}, b=\Sigma_{|n|=\nu(b)} b_{n}$ where $a_{m}=c_{m} y^{m}, b_{n}=d_{n} y^{n}, y^{m}=y_{1}^{m} 1 \ldots$ $y_{k}^{m_{k}} ; c_{m}, d_{n} \in J$. Now let $u \rightarrow \rho(u)$ be the usual valuation of an element $u \in H$ (the case where $\mathrm{i}=0$ ). Then one has $\rho(a)=\nu(a)+\alpha(a)$ and $\rho(b)=\nu(b)+\alpha(b)$ where for any $u \in H$ one puts $\alpha(u)=\max _{r} \rho\left(e_{r}\right)$ where $e_{r} \in J$ is such that $u=$ $\Sigma e_{r} y^{r}$. But now if $v=\Sigma c_{m} d_{n} y^{m+n}$ where the sum is over all pairs $(m, n)$ such that $|m|=\nu(a), \rho\left(c_{m}\right)=\alpha(a),|n|=\nu(b), \rho\left(d_{n}\right)=\alpha(b)$ then clearly

$$
a b-v \in H_{(\rho(a)+\rho(b)-1)} .
$$

On the other hand, since $\rho(a b)=\rho(a)+\rho(b)$ it follows that $v \notin H_{\rho(a)+\rho(b)-1}$, so that $v$ can be written $v=\Sigma_{|r|=v(a)+\nu(b)} e_{r} y^{r}$ where $\alpha(v)=\alpha(a)+\alpha(b)$. On the other hand by (7.2) one has, for some $f_{s} \in J, a b-v=\Sigma_{|s| \leqslant \nu(a)+\nu(b)} f_{s} y^{s}$ and $\rho\left(f_{s}\right)<\alpha(a)+\alpha(b)$ for $|s|=\nu(a)+\nu(b)$. Thus one cannot have $e_{r}+f_{r}=0$ for all $r$ where $|r|=\nu(a)+\nu(b)$. This implies $\nu(a b)=\nu(a)+\nu(b)$. Q.E.D. 
We can identify the universal enveloping algebra of the direct sum $\mathfrak{l}_{\mathbf{C}}+\mathfrak{a}_{\mathbf{C}}$ with $K \otimes A$. Since $t_{c}$ is a subalgebra in $t_{c} \oplus a_{c}$ it defines a valuation $\nu_{0}$ on $K \otimes A$. Now $A$ is graded $A=\bigoplus A_{i}$. If $v_{0}(u)=d, u \in K \otimes A$ then there exists a unique $u_{d} \in K \otimes A_{d}$ such that

$$
v_{0}\left(u-u_{d}\right)<d
$$

Since $K \otimes A$ satisfies the Ore condition and is an integral domain, $\nu_{0}$ extends to the quotient division ring $Q(K \otimes A)$.

We let $\nu$ be the valuation on $G$ and on $Q(G)$ its quotient division ring, defined also by $t_{C}$.

A proof of the following proposition can be found in Lepowsky [9]. We first recall that the map $P: G \rightarrow K \otimes A$ was the projection defined by the decomposition $G=K \otimes A \oplus G n_{\mathrm{C}}$. Let $\lambda: S\left(g_{\mathrm{C}}\right) \rightarrow G$ denote the symmetrization mapping. We note that $\lambda$ is defined on $S\left(\mathfrak{p}_{\mathrm{C}}\right)$ by regarding $S\left(\mathfrak{p}_{\mathrm{C}}\right) \subset S\left(\mathbb{8}_{\mathrm{C}}\right)$. Let $q$ be the orthogonal complement of $\mathfrak{a}$ in $\mathfrak{p}$ with respect to the Killing form of $\mathfrak{q}$, and let $\mathfrak{q}_{\mathrm{C}} \subset \mathfrak{p}_{\mathrm{c}}$ be the complexification of $\mathfrak{q}$. Then $S\left(\mathfrak{p}_{\mathrm{C}}\right)=S\left(\mathfrak{a}_{\mathrm{c}}\right) \oplus \mathfrak{q}_{\mathrm{C}} S\left(\mathfrak{p}_{\mathrm{C}}\right)$, so that

$$
G=(K \otimes A) \oplus\left(K \otimes \lambda\left(\mathfrak{q}_{c} S\left(\mathfrak{p}_{c}\right)\right)\right) .
$$

Let $F: G \rightarrow K \otimes A$ denote the projection onto the first summand in this decomposition.

Proposition 7.2. (i) If $u \in G^{K}$ then $v(u)=\nu_{0}(F(u)$ ). (ii) If $0 \neq u \in G$ then $v_{0}(P(u)-F(u))<v(u)$.

Corollary 7.3. If $u \in G^{K}$ then $v(u)=\nu_{0}(P(u))$.

Proor. If $u \in G^{K}$ we have $\nu_{0}(P(u)-F(u))<\nu_{0}(F(u))$; hence the leading term of $F(u)$ is equal to the leading term of $P(u)$, and therefore $\nu_{0}(P(u))=$ $\nu_{0}(F(u))=\nu(u)$.

Now let $\delta: 8_{C} \rightarrow 8_{C}^{\prime}$ be the isomorphism defined by the Killing form of $8_{\mathrm{C}}$. We may extend $\delta$ to an algebra, $G_{\mathrm{C}}$-isomorphism of symmetric algebras $\delta$ : $S\left(8_{\mathrm{C}}\right) \rightarrow S\left(8_{\mathrm{C}}^{\prime}\right), G_{\mathrm{C}}$ being the adjoint group of $g_{\mathrm{C}}$.

Let $a \in\left(S^{n-l}\left(g_{\mathrm{C}}^{\prime}\right)\right)^{G_{\mathrm{C}}}$ be as at the end of $\S$ and let $\alpha=\delta^{-1}(a)$ so that $\alpha \in\left(S^{n-l}\left(g_{\mathbf{C}}\right)\right)^{G} \mathbf{C}$. Finally $\lambda: S\left(g_{C}\right) \rightarrow G$ is a $G_{\mathbf{C}}$-linear isomorphism and we put $\gamma=\lambda(\alpha)$ so that $\gamma \in$ Cent $G \subset G^{K}$. Now $\gamma_{0}=P(\gamma) \in B \subset K^{M} \otimes A$ (cf. $\S 4)$ and hence $P$ induces an antihomomorphism $P_{\gamma}: G_{\gamma}^{K} \rightarrow B_{\gamma_{0}}$. Note that $\gamma_{0} \in$ Center $K^{M} \otimes A$ since one easily has $\gamma_{0} \in M \otimes A$ where $M$ is the enveloping algebra of the Lie algebra of $M$. Clearly $P$ is compatible with valuations (see Corollary 7.3). Therefore $P_{\gamma}$ extends to a map $P_{\Gamma}$ of the respective completions $G_{\Gamma}^{K}$ and $B_{I_{0}}$. We have

Throrem 7.4. The map $P_{\Gamma}: G_{\Gamma}^{K} \rightarrow B_{\Gamma_{0}}$ is a surjective anti-isomorphism. 
Proof. To prove the theorem it is sufficient to show that given $u_{0} \in B_{\gamma_{0}}$ there exists $u \in G_{\gamma}^{K}$ such that

(a) $\nu_{0}\left(u_{0}\right)=v(u)$, and

(b) $\nu_{0}\left(u_{0}-P_{\gamma}(u)\right)<\nu_{0}\left(u_{0}\right)$.

In fact it suffices only to prove (b) since (b) $\Rightarrow(a)$. This is clear since, by writing $P_{\gamma}(u)=u_{0}-\left(u_{0}-P_{\gamma}(u)\right)$ and $u_{0}=\left(u_{0}-P_{\gamma}(u)\right)+P_{\gamma}(u)$, (2) implies that $\nu_{0}\left(u_{0}\right)=v_{0}\left(P_{\gamma}(u)\right)$. But $\nu_{0}\left(P_{\gamma} u\right)=v(u)$.

Next note that we may assume that $u_{0} \in B$. Indeed assume the theorem is true in this case. Write $u_{0}=f_{0} / \gamma_{0}^{i}$ where $f_{0} \in B$. But then there exists $f \in$ $G_{\gamma}^{K}$ such that $\nu_{0}\left(f_{0}-P_{\gamma} f\right)<\nu_{0}\left(f_{0}\right)$. Hence

$$
\nu_{0}\left(u_{0}-P_{\gamma}\left(f / \gamma^{i}\right)\right)=\nu_{0}\left(f_{0}-P_{\gamma}(f)\right)-\nu_{0}\left(\gamma_{0}^{i}\right)<\nu_{0}\left(f_{0}\right)-\nu_{0}\left(\gamma_{0}^{i}\right)=\nu_{0}\left(u_{0}\right) \text {. }
$$

Thus we assume $u_{0}=f_{0} \in B$.

Let $G_{(n)}$ be the usual filtration of $G$ and $B_{(n)}$ be the usual filtration for $B$. (See beginning of this section.) Now let $\sigma^{n}: G_{(n)} \rightarrow S^{n}\left(g_{C}^{\prime}\right)$ be the linear map defined by composing $\lambda^{-1}: G_{(n)} \rightarrow S_{(n)}\left(8_{\mathrm{C}}\right)=\sum_{j=0}^{n} S^{j}\left(8_{\mathrm{C}}\right)$ with $\delta: S_{(n)}\left(8_{\mathrm{C}}\right)$ $\rightarrow S_{(n)}\left(g_{\mathrm{C}}^{\prime}\right)$ and then with the projection $S_{(n)}\left(g_{\mathrm{C}}^{\prime}\right) \rightarrow S^{n}\left(g_{\mathrm{C}}^{\prime}\right)$. It is clear that $\sigma^{n}$ is a $G_{\mathrm{C}}$-linear map. Let $\sigma_{0}^{n}:(K \otimes A)_{(n)} \rightarrow S^{n}\left(\left(\mathfrak{l}_{\mathrm{C}} \oplus a_{\mathrm{C}}\right)^{\prime}\right)$ be defined similarly so that $\sigma_{0}^{n}$ is a $K_{\mathrm{C}}$-linear map. It then follows easily from the definition of the map $F: G \rightarrow K \otimes A$ and the Birkhoff-Witt theorem that one has a commutative diagram

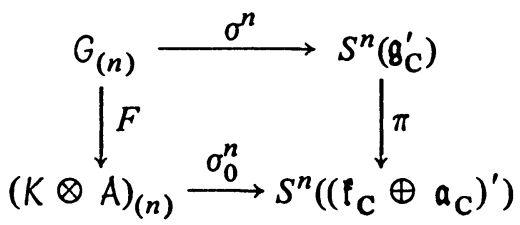

where, recall, $\pi: S\left(g_{\mathrm{C}}^{\prime}\right) \rightarrow S\left(\left(\mathfrak{f}_{\mathrm{C}} \oplus \mathrm{a}_{\mathrm{C}}\right)^{\prime}\right)$ is the restriction map $\varphi \rightarrow \varphi \mid\left(\mathfrak{f}_{\mathrm{C}} \oplus \mathrm{a}_{\mathrm{C}}\right)$ for $\varphi \in S\left(g_{\mathrm{C}}^{\prime}\right)$.

Now let $\rho$ be the usual valuation on $G$. Thus if $u \in G$ then $\rho(u)=-\infty$ if $u=0$, otherwise $\rho(u)$ is the least $n \in Z_{+}$such that $u \in G_{(n)}$. Note that if $u \in$ $G_{(n)}$ then $\lambda^{n}(u) \neq 0$ if and only if $\rho(u)=n$. One defines the valuation $\rho_{0}$ on $K \otimes A$ similarly. Now since $\pi$ is injective on $S\left(g_{C}^{\prime}\right)^{K} \mathbf{C}$ it then follows from (7.4) that, for any $u \in G^{K}, \rho(u)=\rho_{0}(F u)$.

The proof of Theorem 7.4 will follow easily from

Lemma 7.5. For any $0 \neq f_{0} \in B$ there exists $j \in Z_{+}$and $w \in G^{K}$ such that $\nu_{0}\left(f_{0} \gamma_{0}^{j}-P(w)\right)<\nu_{0}\left(f_{0} \gamma_{0}^{j}\right)$. (Note that this implies $\nu_{0}\left(f_{0} \gamma_{0}^{j}\right)=\nu_{0}(P(w))$ and since $\left(\right.$ Corollary 7.3) $\nu_{0}(P(w))=\nu(w)$ this also implies $\nu_{0}\left(f_{0} \gamma_{0}^{j}\right)=\nu(w)$.)

Proof of Lemma 7.5. For any $0 \neq x \in K \otimes A$ let $\tilde{x} \in K \otimes A$ be the unique element defined so that if $\nu_{0}(x)=d$ then $\tilde{x} \in K \otimes A_{d}$ and $\nu_{0}(x-\tilde{x})<d$. 
Clearly $\tilde{x} \neq 0$ and $\rho_{0}(\tilde{x}) \geqslant \nu_{0}(\tilde{x})=\nu_{0}(x)=d$. Put $\beta(x)=\rho_{0}(\tilde{x})-\nu_{0}(x)$.

We will prove the lemma by induction on $\beta\left(f_{0}\right)$. Let $\tau: S\left(g_{C}^{\prime}\right) \rightarrow G$ be the

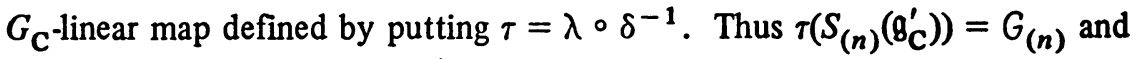
$\sigma^{n} \circ \tau$ is the identity on $S^{n}\left(g_{C}^{\prime}\right)$.

Now assume $\beta\left(f_{0}\right)=0$. Thus $\tilde{f_{0}} \in A_{d}$ where $d=\nu_{0}\left(f_{0}\right)$. But then, by Theorem 4.5, $\widetilde{f}_{0}$ and hence $\sigma_{0}^{d}\left(\widetilde{f}_{0}\right) \in S^{d}\left(a_{\mathrm{c}}^{\prime}\right)$ is Weyl group invariant. Thus there exists (see e.g. [16, Theorem 6.10]) $\xi \in S^{d}\left(\mathfrak{p}_{\mathrm{C}}^{\prime}\right)^{K_{\mathbf{C}}} \subseteq S^{d}\left(\boldsymbol{g}_{\mathrm{C}}^{\prime}\right)^{K_{\mathbf{C}}}$ such that $\pi(\xi)=\sigma_{0}^{d}\left(\widetilde{f}_{0}\right)$. But then if $w=\tau \xi$ one has $w \in G^{K}$. But by (7.4) one has $\sigma_{0}^{d}(F(w))=\sigma_{0}^{d}\left(\tilde{f}_{0}\right)$. Thus $\rho_{0}\left(\tilde{f}_{0}-F(w)\right)<d$. But $v_{0}\left(\tilde{f}_{0}-F(w)\right) \leqslant$ $\rho_{0}\left(\widetilde{f}_{0}-F(w)\right)$ and $\rho_{0}\left(\tilde{f}_{0}\right)=\nu_{0}\left(\tilde{f}_{0}\right)=d$. Thus $\nu_{0}\left(\widetilde{f}_{0}-F(w)\right)<v_{0}\left(\widetilde{f_{0}}\right)=d$. But then $\nu_{0}\left(f_{0}-F(w)\right)<d=\nu_{0}\left(f_{0}\right)$. Hence $\nu_{0}(F(w))=d$. But then $v_{0}(F(w)-P(w))<d$ by Proposition 7.2. Thus $v_{0}\left(f_{0}-P(w)\right)<d$ proving the lemma for $\beta\left(f_{0}\right)=0$.

Now assume $\beta\left(f_{0}\right)>0$ and assume the lemma is true for smaller values. Again let $d=\nu_{0}\left(\tilde{f}_{0}\right)=\nu_{0}\left(f_{0}\right)$.

Now put $m=\rho_{0}\left(\widetilde{f}_{0}\right)$ so that $m-d=\beta\left(f_{0}\right)$. But by Theorem $4.50 \neq$ $\sigma_{0}^{m}\left(\widetilde{f}_{0}\right) \in S^{m}\left(\left(\ell_{\mathrm{C}} \oplus a_{\mathrm{C}}\right)^{\prime}\right)$ is Weyl group invariant. But then by Theorem 6.1 there exist $i \in Z_{+}$and $\psi \in S^{r}\left(g_{C}^{\prime}\right)^{K} \mathbf{C}$ where $r=m+i(n-l)$ such that $\psi \mid \mathfrak{l}_{\mathrm{C}} \oplus \mathrm{a}_{\mathrm{C}}=\sigma_{0}^{m}\left(\tilde{f}_{0}\right) b_{0}^{i}$. Furthermore since $\sigma_{0}^{m}\left(\tilde{f}_{0}\right) b_{0}^{i} \in S\left(\mathfrak{l}_{\mathrm{C}}^{\prime}\right) \otimes S^{p}\left(a_{\mathrm{C}}^{\prime}\right)$ where $p=d+i(n-l)$ it follows from the injectivity of $\pi \mid S\left(\mathfrak{g}_{\mathrm{C}}^{\prime}\right)^{K} \mathbf{C}$ that $\psi \in S\left(\mathfrak{l}_{\mathrm{C}}^{\prime}\right) \otimes$ $S^{p}\left(p_{C}^{\prime}\right)$. It follows therefore, if we put $u=\tau(\psi) \in G^{K}$, that $\nu(u) \leqslant p$. On the other hand by (7.4) one has

$$
\sigma_{0}^{r}(F(u))=\sigma_{0}^{m}\left(f_{0}\right) b_{0}^{i} \neq 0 .
$$

But since $0 \neq \sigma_{0}^{r}(F(u)) \in S\left(\mathfrak{l}_{\mathrm{C}}\right) \otimes S^{p}\left(\mathfrak{a}_{\mathrm{C}}^{\prime}\right)$ it follows that $\nu_{0}(F(u)) \geqslant p$. Thus

$$
\nu_{0}(F(u))=v(u)=p
$$

since $\nu(u)=\nu_{0}(F(u))$.

Now by definition $\gamma_{0}=P(\gamma)$ and $\gamma=\tau(a)$ where $a \in S^{n-l}\left(g_{C}^{\prime}\right)$ is defined as in $\S 5$. Obviously, then $\rho(\gamma)=n-l$ so that $\rho_{0}\left(\gamma_{0}\right) \leqslant n-l$.

Now Proposition 7.2 clearly implies that for any $v \in G^{K}$ one has

$$
\widetilde{P(v)}=\widetilde{F(v)} \text {. }
$$

Now we assert that $\rho_{0}\left(\tilde{\gamma}_{0}\right)=n-l$ and in fact

$$
\sigma_{0}^{n-l}\left(\tilde{\gamma}_{0}\right)=b_{0} \in S^{n-l}\left(a_{c}^{\prime}\right) \text {. }
$$

Indeed by definition $a \mid a_{\mathrm{C}}^{\prime}=b_{0}$. But then if $a_{0}=\pi(a)$ one has that $a_{0}=$ $b_{0}+a_{1}$ where $a_{1} \in S\left(\mathrm{f}_{\mathrm{C}}^{\prime}\right) \otimes S_{(n-l-1)}\left(\mathrm{a}_{\mathrm{C}}^{\prime}\right)$. But by (7.4) $\sigma_{0}^{n-l}(F(\gamma))=a_{0}$ and hence $\sigma_{0}^{n-l}(\widetilde{F(\gamma)})=b_{0}$. Then by (7.7) $\sigma_{0}^{n-l} .\left(\widetilde{\gamma}_{0}\right)=b_{0}$ establishing (7.8) and hence also that $\rho_{0}\left(\tilde{\gamma}_{0}\right)=n-l$. This implies $\rho_{0}\left(\gamma_{0}\right)=n-l$ since $n-l=\rho(\gamma) \geqslant$ 
$\rho_{0}\left(\gamma_{0}\right) \geqslant \rho_{0}\left(\tilde{\gamma}_{0}\right)=n-l$. Note that (7.8) also implies that $\nu_{0}\left(\gamma_{0}\right)=\nu_{0}\left(\tilde{\gamma}_{0}\right)=$ $n-l$.

Now for any $w, v \in K \otimes A$ note that $\widetilde{w v}=\tilde{w} \tilde{v}$. Hence $\widetilde{f_{0} \gamma_{0}^{i}}=\widetilde{f}_{0} \widetilde{\gamma}_{0}^{i}$. Thus since $\nu_{0}(x)=\nu_{0}(\tilde{x})$ for $0 \neq x \in K \otimes A$ this implies that

$$
\nu_{0}\left(f_{0} \gamma_{0}^{i}\right)=p \text {. }
$$

On the other hand by (7.6) and Corollary 7.3, $\nu_{0}(P u)=p$. If $\nu_{0}\left(f_{0} \gamma_{0}^{i}-P u\right)$ $<p$ we are done. Assume therefore, that $\nu_{0}\left(f_{0} \gamma_{0}^{i}-P u\right)=p$. Thus $\widetilde{f_{0} \gamma_{0}^{i}}$ and $\widetilde{P u}$ are distinct elements of $K \otimes A_{p}$ and hence if $x=f_{0} \gamma_{0}^{i}-P(u)$ one has $x \in B$ and $\tilde{x}=\widetilde{f_{0} \gamma_{0}^{i}}-\widetilde{P(u)} \in K \otimes A_{p}$. But $\rho_{0}\left(\widetilde{f_{0} \gamma_{0}^{i}}\right)=\rho_{0}\left(\widetilde{f}_{0}\right) \rho_{0}\left(\tilde{\gamma}_{0}\right)^{i}=r$. However by (7.5) $r=\rho(u) \geqslant \rho_{0}(\widetilde{P(u)})$. Thus $r \geqslant \rho_{0}(\widetilde{x})$. On the other hand one has $\sigma_{0}^{r}\left(\widetilde{f_{0} \gamma_{0}^{i}}\right)=\sigma_{0}^{r}\left(\widetilde{f}_{0} \widetilde{\gamma}_{0}^{i}\right)$. But then

$$
\sigma_{0}^{r}\left(\widetilde{f_{0} \gamma_{0}^{i}}\right)=\sigma_{0}^{m}\left(\widetilde{f}_{0}\right) b_{0}^{i}
$$

by (7.8) since if $y \in(K \otimes A)_{(s)}$ and $z \in(K \otimes A)_{(t)}$ then

$$
\sigma_{0}^{s+t}(y z)=\sigma_{0}^{s}(y) \sigma_{0}^{t}(z)
$$

Now $\sigma_{0}^{r}(F(u))=\sigma_{0}^{m}\left(\tilde{f_{0}}\right) b_{0}^{i}$ by (7.5). We assert that $\sigma_{0}^{r}(F(u))=\sigma_{0}^{r}(F(\tilde{u}))$. Indeed $\sigma_{0}^{r}(\widetilde{F(u)}) \in S\left(\mathrm{f}_{\mathrm{C}}^{\prime}\right) \otimes S^{p}\left(\mathrm{~g}_{\mathrm{C}}^{\prime}\right)$ and $\sigma_{0}^{r}(\widetilde{F(u)}) \in S\left(\mathrm{f}_{\mathrm{C}}^{\prime}\right) \otimes S^{p}\left(\mathrm{a}_{\mathrm{C}}^{\prime}\right)$ since $\nu_{0}(F(u))=p$ by (7.6). However one necessarily has $\sigma_{0}^{r}(F(u))-\widetilde{F}(u) \in$ $S\left(\mathrm{f}_{\mathrm{C}}^{\prime}\right) \otimes S_{(p-1)}\left(\mathrm{a}_{\mathrm{C}}^{\prime}\right)$ since $v_{0}(F(u)-\widetilde{F}(u))<p$. Thus $\sigma_{0}^{r}(\widetilde{F}(u))=\sigma_{0}^{r}(F(u))=$ $\sigma_{0}^{m}\left(\widetilde{f}_{0}\right) b_{0}^{i}$. But $\sigma_{0}^{r}(\widetilde{P(u)})=\sigma_{0}^{r}(\widetilde{F(u)})$ by (7.7). Thus recalling (7.10), one has $\sigma_{0}^{r}(\tilde{x})=0$ so that $\rho_{0}(\tilde{x})<r$. But then $\beta(x)=\rho_{0}(\tilde{x})-p<r-p=m-d$. The induction assumption then applies to $x$ so that for some $k \in Z_{+}$there exists $v \in G^{K}$ such that $\nu_{0}\left(x \gamma_{0}^{k}-P(v)\right)<\nu_{0}\left(x \gamma_{0}^{k}\right)$. But $x \gamma_{0}^{k}=\left(f_{0} \gamma_{0}^{i}-P(u)\right) \gamma_{0}^{k}=$ $f_{0} \gamma_{0}^{j}-P\left(\gamma^{k} u\right)$ where $j=k+i$. Now put $w=v+\gamma^{k} u \in G^{K}$. Then $\nu_{0}\left(f_{0} \gamma_{0}^{j}-P(w)\right)<\nu_{0}\left(x \gamma_{0}^{k}\right)=p+k(n-l)=d+j(n-l)=\nu_{0}\left(f_{0} \gamma_{0}^{j}\right)$. Q.E.D.

To finish the proof of the theorem let $0 \neq f_{0} \in B$ and let $j \in Z_{+}$and $w \in$ $G^{K}$ be given by Lemma 7.5. Now put $f=w / \gamma^{j}$. Then

$$
\begin{aligned}
& \nu_{0}\left(f_{0}-P_{\gamma}(f)\right)=\nu_{0}\left(f_{0} \gamma_{0}^{j}-P_{\gamma}(f) \gamma_{0}^{j}\right)-j(n-l) \\
& \quad=\nu_{0}\left(f_{0} \gamma_{0}^{j}-P(w)\right)-j(n-l)<\nu_{0}\left(f_{0} \gamma_{0}^{j}\right)-j(n-l)=\nu_{0}\left(f_{0}\right) \text {. Q.E.D. }
\end{aligned}
$$

\section{REFERENCES}

1. L. Cohn, Some theorems on C-functions (to appear).

2. J. Dieudonné and J. B. Carrell, Invariant theory, old and new, Advances in Math. 4 (1970), 1-80. MR 41 \#186.

3. J. Dixmier, Algèbres enveloppantes, Gauthier-Villars, Paris, 1974.

4. A. Erdélyi, Asymptotic expansions, Dover, New York, 1956. MR 17 \#1202.

5. A. Knapp and E. Stein, Intertwining operators for semi-simple Lie groups, Ann. of Math. (2) 93 (1971), 489-578. 
6. B. Kostant, On the existence and irreducibility of certain series of representations, 1971 Summer School in Math., edited by I. M. Gelfand.

7. B. Kostant and S. Rallis, Orbits and representations associated with symmetric spaces, Amer. J. Math. 93 (1971), 753-809. MR 47 \#399.

8. R. A. Kunze and E. M. Stein, Uniformly bounded representations. III. Intertwining operators for the principal series on semisimple groups, Amer. J. Math. 89 (1967), 385-442. MR 38 \#269.

9. J. Lepowsky, Algebraic results on representations of semi-simple Lie groups, Trans. Amer. Math. Soc. 176 (1973), 1-44.

10. J. Lepowsky and G. W. McCollum, On the determination of irreducible modules by restriction to a subalgebra, Trans. Amer. Math. Soc. 176 (1973), 45-57. MR 48 \#2201.

11. W. Magnus, F. Oberbettinger and R. P. Soni, Formulas and theorems for the special functions of mathematical physics, 3rd ed., Die Grundlehren der math. Wissenschaften, Band 52, Springer-Verlag, New York, 1966. MR 38 \#1291.

12. G. Schiffmann, Intégrales d'entrelacement et fonctions de Whittaker, Bull. Soc. Math. France 99 (1971), 3-72. MR $47 \# 400$.

13. N. Wallach, Harmonic analysis on homogeneous spaces, Dekker, New York, 1973.

14. G. Warner, Harmonic analysis on semi-simple Lie groups, I, Springer-Verlag, New York, 1972.

15. D. Mumford, Introduction to algebraic geometry, Harvard Univ. Notes.

16. S. Helgason, Differential geometry and symmetric spaces, Pure and Appl. Math., vol. 12, Academic Press, New York, 1962. MR 26 \#2986.

DEPARTMENT OF MATHEMATICS, MASSACHUSETTS INSTITUTE OF TECHNOLOGY, CAMBRIDGE, MASSACHUSETTS 02139 